\title{
THE CRITERIA OF CHECKING HYPOTHESES OF QUANTUM STATES OF QUANTUM PHYSICAL SYSTEMS
}

\section{MALKHAZ MUMLADZE and ZURAB ZERAKIDZE}

Gori University

Georgia

e-mail: mmumladze@mail.ru

\begin{abstract}
In this article, we define and build the criteria for the testing of hypotheses in the physical quantum systems.

For each quantum state, we construct the measure in the space $\left(P_{U}, S\right)$, where $P_{U}$ is the space of the pure states of the quantum system, $S$ is the Borel $\sigma$-algebra in $P_{U}$. The criteria for the checking hypotheses of the quantum states we call a measurable map $\delta:\left(P_{U}, S\right) \rightarrow\left(2^{\mathfrak{I}}, \Omega\right)$, where $\mathfrak{I}$ is the space of the hypothetical states with the topological structure induced by topological structure in the space quantum states $\mathfrak{I}_{U}, 2^{\mathfrak{J}}$ is the space of closed subsets of $\mathfrak{I}$ with the exponential topological structure, and $\Omega$ is the Borel $\sigma$-algebra subsets in $2^{\mathfrak{J}}$.
\end{abstract}

2010 Mathematics Subject Classification: 62P35, 28A60, 81Q99.

Keywords and phrases: quantum physical system, state, pure state, observable, measure, criteria of checking hypotheses.

Received December 6, 2015

(C) 2015 Scientific Advances Publishers 


\section{Quantum Physical Systems}

As known [1] a quantum physical system can represented by a couple $(U, \mathfrak{I})$, where $U$ is some $C^{*}$-algebra which Hermit's the elements that are called observables and some set $\mathfrak{I}$ of positive functionals with norm one and called the quantum states of this physical system [2]. We say what the functional $\omega_{1}$ majorizes functional $\omega_{2}$ if $\omega_{1}-\omega_{2}$ is a positive functional. The state $\omega$ quantum physical system is called the pure state if it majorizes only functional type $\lambda \omega, 0 \leq \lambda \leq 1$ [2].

Denote by $P_{U}$ the set of pure states on $C^{*}$-algebra $U$.

In the set of all linear continue functional on $C^{*}$-algebra $U$, we have topological structure which called as weakly topological structure [2] and which defined by pre basis:

$$
V\left(\omega, u_{1}, u_{2}, \ldots, u_{n}\right)=\left\{\omega^{\prime} \in U^{*}|| \omega\left(u_{i}\right)-\omega^{\prime}\left(u_{i}\right) \mid<\varepsilon, \quad i=1,2, \ldots, n\right\},
$$

where $\omega, \omega^{\prime} \in U^{*}, u_{i} \in U$, accordingly this in the set $P_{U}$, we have the topological structure induced from this topological structure.

Denote by $\mathfrak{R}$ the set of Hermit's elements of $U C^{*}$-algebra.

Lemma. Every linear functional on the $U C^{*}$-algebra uniquely will be defined by its values on Banach subspace of Hermit's elements.

Proof. It's known [2] that every element $u$ of $C^{*}$-algebra $U$ uniquely represented as $u_{1}+i u_{2}$, where $u_{1}$ and $u_{2}$ are Hermitian. The lemma is proved.

Every a Hermit's element $u \in \mathfrak{R}$ in the $C^{*}$-algebra $U$ any can be represented by integral

$$
u=\int_{-\infty}^{\infty} \lambda d p_{\lambda}^{u}
$$

where $\left\{p_{\lambda}^{u}\right\}_{\lambda \in R}\left(p_{\lambda}^{u}\right)^{2}=p_{\lambda}^{u}$ projectors and represents the partition of unity of Hermit's element $u \in \mathfrak{R}$ [4]. 
Correspond to projector $p_{\alpha}^{2}=p_{\alpha} \in U$ the family $\left\{p_{\lambda}^{p_{\alpha}}\right\}_{\lambda \in R}$ of elements of the $C^{*}$-algebra $U$ which has the condition: $p_{\lambda}^{p_{\alpha}}=0$, if $\lambda<1, p_{\lambda}^{p_{\alpha}}=p_{\alpha}$, if $\lambda=1$ and $p_{\lambda}^{p_{\alpha}}=E$, if $\lambda>1$, where $E$ is the unit element in the algebra $U$. It is clear that

$$
p_{\alpha}=\int_{-\infty}^{\infty} \lambda d p_{\lambda}^{p_{\alpha}}
$$

If $u \in U$, then by the proved lemma $u=u_{1}+i u_{2}$, where $u_{1}$ and $u_{2}$ are Hermitian elements. The representation such $u$ will be

$$
u=u_{1}+i u_{2}=\int_{-\infty}^{\infty} \lambda d p_{\lambda}^{u_{1}}+i \int_{-\infty}^{\infty} \lambda d p_{\lambda}^{u_{1}}
$$

Obviously, if $\omega$ is some linear continue functional on $C^{*}$-algebra $U$, then from lemma and from the last equality we will have

$$
\omega(u)=\omega\left(u_{1}+i u_{2}\right)=\omega\left(\int_{-\infty}^{\infty} \lambda d p_{\lambda}^{u_{1}}\right)+\omega\left(\int_{-\infty}^{\infty} \lambda d p_{\lambda}^{u_{2}}\right)=\int_{-\infty}^{\infty} \lambda d \omega\left(p_{\lambda}^{u_{1}}\right)+i \int_{-\infty}^{\infty} \lambda d \omega\left(p_{\lambda}^{u_{2}}\right),
$$

where $u_{1}$ and $u_{2}$ are Hermit's elements.

Let $\left\{p_{\alpha}\right\}$ be the set of all one dimensional projectors on $C^{*}$-algebra $U$ and $\omega$ be pure state, then from lemma and the last equality follows that this state has the non zero meaning only on some projector $p_{\alpha} \in\left\{p_{\alpha}\right\}$ and the meaning 0 on the other one dimensional projectors. Otherwise, we can always construct such functional which will not have the type $\lambda \omega, 0 \leq \lambda \leq 1$ and well majorized by the pure state $\omega$. So how, if functional $\omega$ is a pure state, then $\omega\left(u^{2}\right) \geq 0$ for all Hermit's elements $u$. Let $\omega\left(p_{\alpha^{\prime}}\right) \neq 0$ and $\omega_{\alpha^{\prime}}$ such functional, which has the non zero meaning $\varepsilon$ only on some projector $p_{\alpha^{\prime}} \in\left\{p_{\alpha}\right\}$ and the meaning 0 on the other one 
dimensional projectors. It is clear, if we take $\varepsilon$ sufficiently small, then we can achieve, that for every $u \in \mathfrak{R}$ will have place inequality

$$
\left(\omega-\omega_{\alpha^{\prime}}\right)\left(u^{2}\right)=\int_{-\infty}^{\infty} \lambda^{2} d\left(\omega-\omega_{\alpha^{\prime}}\right)\left(p_{\lambda}^{u}\right) \geq 0 .
$$

It means, that the pure state $\omega$ majorize the functional $\omega_{\alpha^{\prime}}$ which does not have the type $\lambda \omega, 0 \leq \lambda \leq 1$, but this is impossible. It follows that the pure states are such functional which satisfy the condition $\omega_{\alpha}\left(p_{\beta}\right)=\delta^{\alpha \beta}$.

An integral representation of Hermit's elements follows that for the pure states $\omega_{\alpha}$ have place the equality $\omega_{\alpha}(u)=\lambda_{\alpha}^{u}$, where $\lambda_{\alpha}^{u}$ is some element of spectrum of Hermit's element $u \in \mathfrak{R}$. It gives opportunity identify every pre state with the set of number $\left\{\lambda_{\alpha}^{u}\right\}_{u \in \mathfrak{R}}$, where $p_{\alpha}(u)=\lambda_{\alpha}^{u}$. Consider the Tikhonov's product $\sum=\underset{u \in \Re}{\otimes} \sigma_{u}$, where $\sigma_{u} \subset R$ spectrum of element $u \in \mathfrak{R}$.

It is clear, $P_{U} \subset \sum$, because $P_{U}$ the set of such elements in product $\Sigma=\underset{u \in \mathfrak{R}}{\otimes} \sigma_{u}$ which represents linear continue maps with respect to the topological structure in $\mathfrak{R}$, which is defined by the norm:

$P_{U}=\left\{\omega_{\alpha}: \mathfrak{R} \rightarrow \underset{u \in \mathfrak{R}}{\cup} \sigma_{u} \mid \omega_{\alpha}(u)=\lambda_{\alpha}^{u}, \omega_{\alpha}\left(k_{1} u_{1}+k_{2} u_{2}\right)=k_{1} \omega_{\alpha}\left(u_{1}\right)+k_{2} \omega_{\alpha}\left(u_{2}\right)\right\}$.

Consequently, in the set $P_{U}$, we have $\sum=\underset{u \in \mathfrak{R}}{\otimes} \sigma_{u}$, induced from Tikhonov's product $\sum=\underset{u \in \mathfrak{R}}{\otimes} \sigma_{u}$ topological structure. Lemma follows, that this topological structure coincides with the induced topological structure from weakly topological structure on set of functionals on $C^{*}$ algebra $U$. We can also identify the set $P_{U}$ with the set of one dimensional projectors $\left\{p_{\alpha}\right\}$. 
Theorem 1. Every state $\omega \in \mathfrak{I}$ in space $P_{U}$ with weakly topological structure, defined on the Borel $\sigma$-algebra of a probability measure $\mu_{\omega}$.

Proof. Let $\omega \in \mathfrak{I}$ be some state. Consider the positive functionals $\nu$ on $C^{*}$-algebra whose value is non zero only on one dimensional projectors $p_{\alpha}$ for which $\omega\left(p_{\alpha}\right) \neq 0$ and in addition $\nu\left(p_{\alpha}\right)=\omega\left(p_{\alpha}\right)$. If such functional exists, then it is clear that $\|\nu\| \leq\|\omega\| \leq 1$.

Let new $\nu_{1}$ and $\nu_{2}$ such functional and in addition if $\nu_{1}\left(p_{\alpha}\right) \neq 0$, then $\nu_{2}\left(p_{\alpha}\right)=0$. As known [2] for the positive functional has place the equality $\left\|\nu_{1}\right\|+\left\|\nu_{2}\right\|=\left\|\nu_{1}+\nu_{2}\right\|$, for our case it follows $\left\|\nu_{1}+\nu_{2}\right\| \leq 1$. Consider the subset $\left\{p_{\alpha_{\beta}}\right\}$ of $P_{U}$, such positive functional $\nu,\|\nu\| \leq 1$, if this exists, which values on the elements of this subset are coincide to corresponding values of the state $\omega$ and the relevance $\mu_{\omega}:\left\{\left\{p_{\alpha_{\beta}}\right\}\right\} \rightarrow R$, which defined by the equality $\mu_{\omega}\left(\left\{p_{\alpha_{\beta}}\right\}\right)=\|\nu\|$. This relevance can not be identified for every subset in $P_{U}$, but it is $\sigma$-additive. Indeed, for functionals $\nu_{1}$ and $\nu_{2}$ whose satisfied the condition: if $\nu_{1}\left(p_{\alpha}\right) \neq 0$, then $\nu_{2}\left(p_{\alpha}\right)=0$, has place equality $\left\|\nu_{1}\right\|+\left\|\nu_{2}\right\|=\left\|\nu_{1}+\nu_{2}\right\|$.

For finite sum $v_{1}+v_{2}+\ldots+v_{n}$, where every $v_{i}$ such that if $\nu_{i}\left(p_{\alpha}\right) \neq 0$, then $\nu_{j}\left(\rho_{\alpha}\right)=0$ when $i \neq j$ also has place the equality

$$
\left\|\nu_{1}\right\|+\left\|\nu_{2}\right\|+\ldots+\left\|\nu_{n}\right\|=\left\|\nu_{1}+\nu_{2}+\ldots+\nu_{n}\right\| \leq 1 .
$$

Let the given series of positive functionals $\nu_{1}+\nu_{2}+\ldots+\nu_{n}+\ldots$, where every $\nu_{i}$ such that if $\nu_{i}\left(p_{\alpha}\right) \neq 0$ then $v_{j}\left(\rho_{\alpha}\right)=0$ when $i \neq j$. This series is converge, otherwise the norm of the functional $\omega \in \mathfrak{I}$ will not be one.

The sum of this series is $\lim _{n \rightarrow \infty}\left(v_{1}+\nu_{2}+\ldots+v_{n}\right)$.

$$
\lim _{n \rightarrow \infty}\left\|\nu_{1}+\nu_{2}+\ldots+\nu_{n}\right\|=\lim _{n \rightarrow \infty}\left(\left\|\nu_{1}\right\|+\left\|\nu_{2}\right\|+\ldots+\left\|\nu_{n}\right\|\right) \leq 1 .
$$


If limit include in norm symbol we obtain equality

$$
\left\|\lim _{n \rightarrow \infty}\left(\nu_{1}+\nu_{2}+\ldots+v_{n}\right)\right\|=\lim _{n \rightarrow \infty}\left(\left\|v_{1}\right\|+\left\|\nu_{2}\right\|+\ldots+\left\|v_{n}\right\|\right) .
$$

It follows that

$$
\left\|\nu_{1}\right\|+\left\|\nu_{2}\right\|+\ldots+\left\|\nu_{n}\right\|+\ldots=\left\|\nu_{1}+\nu_{2}+\ldots+\nu_{n}+\ldots\right\| \leq 1
$$

It means that relevance $\mu_{\omega}:\left\{\left\{p_{\alpha_{\beta}}\right\}\right\} \rightarrow R$ is $\sigma$-additive.

If $\omega \in \mathfrak{I}$ is pure state then the according measure will be the Dirac's measure [2]. In space $P_{U}$ with weakly topological structure the closure of set such points whose elements represents all projectors on which state $\omega \in \mathfrak{I}$ are different from zero is called as support of this state. It is denoted so: Support $\omega$.

Let $\left\{p_{\alpha_{\beta}}\right\}$ be the closed subset in space $P_{U}$ with weakly topological structure. Consider set $\left\{p_{\alpha_{\beta}}\right\} \cap$ Support $\omega$. The functional $\nu$, which coincide to $\omega \in \mathfrak{I}$ on this intersection and is zero on the other one dimensional projectors will be continue, it follows that $v$ is positive [2].

It is clear that $\|\nu\| \leq 1$. It means that the relevance $\mu_{\omega}:\left\{\left\{p_{\alpha_{\beta}}\right\}\right\} \rightarrow R$, $\mu_{\omega}\left(\left\{p_{\alpha_{\beta}}\right\}\right)=\|\nu\|$ is defined for all closed subsets of $P_{U}$ with weakly topological structure.

If $\left\{p_{\alpha_{\beta}}\right\}$ is the closed subset in $P_{U}$ consider $\overline{P_{U} \backslash\left\{p_{\alpha_{\beta}}\right\} \cap \text { Support } \omega}$, there exists such $\nu,\|\nu\| \leq 1$ positive functional that $\mu_{\omega}\left(\left\{p_{\alpha_{\beta}}\right\}\right)=\|\nu\|$. Let $\bar{v}$ be such functional which coincide to $\omega \in \mathfrak{I}$ functional on this intersection and is zero on other one dimensional projectors. This functional is continue and therefore positive. It is clear that $\|\bar{\nu}\|=1-\|\nu\|$. We will assume $\mu_{\omega}\left(\left\{P_{U} \backslash\left\{p_{\alpha_{\beta}}\right\}\right\}\right)=\|\bar{\nu}\|$. It follows that the relevance $\mu_{\omega}:\left\{\left\{p_{\alpha_{\beta}}\right\}\right\} \rightarrow R, \mu_{\omega}\left(\left\{p_{\alpha_{\beta}}\right\}\right)=\|\nu\|$ is also defined for open subsets of $P_{U}$ with weakly topology. So ultimately we built the probability measure on the Borel $\sigma$-algebra of space $P_{U}$ with weakly topological structure. 
The theorem is proved.

We call space $P_{U}$ with weakly topological, as space of quantum physical system. If $C^{*}$-algebra $U$ has a unit, then in the space $U^{*}$ with weakly topological structure the set of al state $\mathfrak{I}$ is convex compact set and represent convex linear combination of pure states $\zeta_{1}, \zeta_{2}, \ldots, \zeta_{n}$ from the set $P_{U}$ :

$$
\omega=k_{1} \zeta_{1}+k_{2} \zeta_{2}+\ldots+k_{n} \zeta_{n}, k_{i} \geq 0, \sum_{i=1}^{n} k_{i}=1
$$

or limit of sequence $\omega_{1}, \omega_{2}, \ldots, \omega_{l}$, where $\omega_{l}=k_{1}^{l} \zeta_{1}^{l}+k_{2}^{l} \zeta_{2}^{l}+\ldots+k_{n_{l}}^{l} \zeta_{n_{l}}^{l}$, $k_{i}^{l} \geq 0, \sum_{i=1}^{n_{l}} k_{i}^{l}=1$ [2]. This means, that elements of set $P_{U}$ are the extreme points of set [2].

Because each state $\omega \in \mathfrak{I}_{u}$ define sprobabiliti measure $\mu_{\omega}$ on couple $\left(P_{U}, S\right)$, where $S$ is borel $C^{*}$-algebra therefore it ease to show, that every $\mu_{\omega}$ represent convex linear combination

$$
\mu_{\omega}=\sum_{i=1}^{n} k_{i} \mu_{\zeta_{i}}, k_{i} \geq 0, \sum_{i=1}^{n} k_{i}=1
$$

of Dirak measures $\mu_{\zeta_{i}}$ where $\xi_{i} \in P_{U}, i=1,2, \ldots, n$ or limit of sequense $\left\{\mu_{\omega_{n}}\right\}_{n \in N}$, where $\mu_{\omega_{n}}=k_{1}^{n} \mu_{\zeta_{1}^{n}}+k_{2}^{n} \mu_{\zeta_{2}^{n}}+\ldots+k_{n_{l l}}^{n} \mu_{\zeta_{l}^{n}}, k_{l}^{n} \geq 0, \sum_{i=1}^{l} k_{i}^{n}=1$ $[2]$.

For every state $\omega \in \mathfrak{I}$, we have $\int_{-\infty}^{\infty} d \omega\left(E_{\lambda}^{u}\right)=1$, therefore it is easy that the value of quantum state on observable $u \in \mathfrak{R}$ is the middle value of this observable. The value $\omega(u) \in R$ is called the middle value of observable $u \in \mathfrak{R}$ of quantum physical system in the state $\omega \in \mathfrak{I}$. 
All told above follows that a quantum physical system is object

$$
\left(U, P_{U}, S, \mu_{\omega}, \omega \in \mathfrak{I}\right),
$$

where $U$ is some $C^{*}$-algebra, Hermit element of which are called observables of this system, $P_{U}$ is the space of quantum system, $S$ is Borel $\sigma$-algebra in $P_{U}$, and $\mu_{\omega}$ is the probability measure defined by state $\omega \in \mathfrak{I}$.

\section{Criteria of Checking Hypotheses of Quantum States of Quantum Physical Systems}

The space of quantum system is location of quantum particles therefore the supports of states can $\omega \in \mathfrak{I}$ covers this space that is $\underset{\omega \in \mathfrak{I}}{\cup} \operatorname{Supp\omega }=P_{U}$.

Let given a quantum physical system

$$
\left(U, P_{U}, S, \mu_{\omega}, \omega \in \mathfrak{I}\right),
$$

and $\Omega$ the Borel's $\sigma$-algebra in the space $2^{\mathfrak{J}}$ of closed subsets in $\mathfrak{I}$ with exponential topological structure, which is induced from weakly topological structure.

Definition 1. We say, that the quantum physical system $\left(U, P_{U}, S\right.$, $\left.\mu_{\omega}, \omega \in \mathfrak{I}\right)$ admits criteria for checking hypotheses of finding in states $\omega \in \mathfrak{I}$ with probability $p \geq \alpha, 0 \leq \alpha \leq 1$, if there exists measurable map

$$
\delta:\left(P_{U}, S\right) \rightarrow\left(2^{\Im}, \Omega\right),
$$

which satisfied condition

$$
\mu_{\omega}\left\{x \in P_{U} \mid \delta(x)=\omega \in \mathfrak{I} \subset 2^{\mathfrak{I}}\right\}=p \geq \alpha,
$$

for all state $\omega \in \mathfrak{I}$. 
Definition 2. The value $p_{\omega}(\delta)=\mu_{\omega}\left\{x \in P_{U} \mid \delta(x) \neq \omega\right\}$ is called the probability of $\omega$-kind of mistake for $\delta$ criteria.

It is easy, that a map $\delta:\left(P_{U}, S\right) \rightarrow\left(2^{\mathfrak{I}}, \Omega\right)$ then, and only then represents consistent criteria for checking hypotheses of finding in the state $\omega \in \mathfrak{J}$ with the probability $p \geq \alpha, 0 \leq \alpha \leq 1$ when the value $p_{\omega}(\delta)=\mu_{\omega}\left\{x \in P_{U} \mid \delta(x) \neq \omega\right\}=q<1-\alpha$ for all states $\omega \in \mathfrak{I}$.

Theorem 2. Let $\mathfrak{I}_{x}$ be the closure in $\mathfrak{I}$ of set of all such states $\omega \in \mathfrak{I}$ which supports contains the point $x \in P_{U}$, then the map

$$
\delta:\left(P_{U}, S\right) \rightarrow\left(2^{\mathfrak{J}}, \Omega\right),
$$

where $\delta(x)=\mathfrak{I}_{x}$, is continue.

Proof. The basis in space $2^{\mathfrak{J}}$ is consists of sets:

$$
V\left(O_{1}, O_{2}, \ldots, O_{k}\right)=\left\{B \in 2^{\mathfrak{J}} \mid B \subset \bigcup_{i=1}^{k} O_{i} ; B \cap O_{i} \neq \varnothing, i=1,2, \ldots, k\right\},
$$

where $O_{1}, O_{2}, \ldots, O_{k}$ is open sets in space $\mathfrak{I}$ with topological structure which induced from weakly topological structure. Let $W$ be an open set in the space $2^{\mathfrak{I}}$. We will show that the set $\delta^{-1}(W)$ is also open. The sets

$$
\begin{aligned}
W= & \bigcup_{\beta} V\left(O_{1}^{\alpha}, O_{2}^{\beta}, \ldots, O_{k_{\beta}}^{\beta}\right) \text {, where } \\
& V\left(O_{1}^{\beta}, O_{1}^{\beta}, \ldots, O_{k_{\beta}}^{\phi}\right)=\left\{B \in 2^{\mathfrak{I}} \mid B \subset \cup_{i=1}^{k_{\beta}} O_{i}^{\beta} ; B \cap O_{i}^{\beta} \neq \varnothing, i=1,2, \ldots, k_{\beta}\right\} .
\end{aligned}
$$

Supports of $\omega \in \mathfrak{I}$ cover $P_{U}$, therefore $\delta^{-1}(W)=\bigcup_{\beta} \bigcup_{i=1}^{k_{\beta}} \bigcup_{\omega_{i}^{\beta} \in O_{i}^{\beta}}$ Support $\omega_{i}^{\beta}$. The set $\bigcup_{\beta} \bigcup_{i=1}^{k_{\beta}} O_{i}^{\beta}$ is opened. If $\delta^{-1}(W)$ is not opened there exists pure state $\omega_{\alpha} \in F r \delta^{-1}(W)$. If $\omega_{\alpha} \in$ Support $\omega$, where $\omega \in \bigcup_{\beta} \bigcup_{i=1}^{k_{\beta}} O_{i}^{\beta}$, then 
$\omega \in \operatorname{Fr} \bigcup_{\beta} \bigcup_{i=1}^{k_{\beta}} O_{i}^{\beta}$, but $\operatorname{Fr} \bigcup_{\beta} \bigcup_{i=1}^{k_{\beta}} O_{i}^{\beta},=\varnothing$, it follows, that we have the contradiction, our assumption of existence $\omega_{\alpha} \in \operatorname{Fr}^{-1}(W)$ is false. It follows that the set $\delta^{-1}(W)$ is opened and $\delta$ continue. The theorem is proved.

Each continue map is measurable, therefore if for $\delta$

$$
\mu_{\omega}\left\{x \in P_{U} \mid \delta(x)=\omega \in \mathfrak{I} \subset 2^{\mathfrak{I}}\right\}=p \geq \alpha,
$$

then quantum physical system admits criteria for checking hypotheses of finding in states $\omega \in \mathfrak{I}$ with probability $p \geq \alpha$.

Definition 3. A quantum physical system is called orthogonal if for each two quantum state $\omega, \omega^{\prime} \in \mathfrak{I}, \omega \neq \omega^{\prime}$ corresponding measures $\mu_{\omega}$, $\mu_{\omega^{\prime}}$ are orthogonal, i.e., there exists subset $N_{\omega^{\prime}} \subset P_{U}$ such that $\mu_{\omega}\left(N_{\omega^{\prime}}\right)=1$ and $\mu_{\omega^{\prime}}\left(P_{U} \backslash N_{\omega^{\prime}}\right)=0$.

Definition 4. Quantum physical system $\left(U, P_{U}, S, \mu_{\omega}, \omega \in \mathfrak{I}\right)$ is called the strongly separable quantum physical system if there exist disjoint family $S$-measurable sets $\left\{X_{\omega}, \omega \in \mathfrak{I}\right\}$ such that the relations are fulfilled: $\forall \omega \in \mathfrak{I} \mu_{\omega}\left(X_{\omega}\right)=1$.

Remark. It's known that every strongly separable quantum physical system are orthogonal [3].

Definition 5. We say, that the quantum physical system $\left(U, P_{U}, S\right.$, $\left.\mu_{\omega}, \omega \in \mathfrak{I}\right)$ admits consistent criteria for checking hypotheses of finding in states $\omega \in \mathfrak{I}$ with probability $p \geq \alpha, 0 \leq \alpha \leq 1$, if there exists measurable map

$$
\delta:\left(P_{U}, S\right) \rightarrow\left(2^{\mathfrak{J}}, \Omega\right),
$$

which satisfied condition

$$
\mu_{\omega}\left\{x \in P_{U} \mid \delta(x)=\omega \in \mathfrak{I} \subset 2^{\mathfrak{I}}\right\}=1,
$$

for all state $\omega \in \mathfrak{I}$. 
Theorem 3. If quantum physical system $\left(U, P_{U}, S, \mu_{\omega}, \omega \in \mathfrak{I}\right)$ is strongly separable, then it admits consistent criteria for checking hypotheses of finding in states $\omega \in \mathfrak{J}$.

Proof. The Theorem 2 tells us that there exists continue map

$$
\delta:\left(P_{U}, S\right) \rightarrow\left(2^{\mathfrak{J}}, \Omega\right),
$$

which defined by formula $\delta(x)=\mathfrak{I}_{x}$. For our case $\mu_{\omega}\left\{x \in P_{U} \mid \delta(x)=\omega\right.$ $\left.\in \mathfrak{I} \subset 2^{\mathfrak{I}}\right\}=1$.

The theorem is proved.

The Theorem 3 and remark, which are made above, follow, that every orthogonal quantum physical system admits consistent criteria for hypotheses.

\section{References}

[1] H. H. Bogolyubov, A. A. Logunov, A. I. Oksak and I. T. Todorov, General Principles of the Quantum Field Theories, Moscow, Nauka, 1987 (in Russian).

[2] U. Bratteli and D. Robinson, Operator Algebras and Quantum Statistical Mechanics, Moscow, Nauka, 1982 (in Russian).

[3] Z. Zerakidze and M Mumladze, Statistical Structures and Consistent Criteria for Checking Hypotheses, LAMBERT Academic Publishing, 2015.

[4] L. A. Liusternirnik and V. I. Sobolev, Elements of Functional Analyses, Moscow, Nauka, 1965 (in Russian). 